\title{
SUSTAINABLE FARMING FOR AGRONOMY UPLIFTMENT USING IOT
}

\author{
Naman Pandey, Aditya Tiwari, Sudhanshu Sharma, Pallerla Sai Teja, Rishabh Jaiswal, Aadarsh Kumar \\ Department of Computer Science and Engineering \\ Vellore Institute of Engineering, Vellore, Tamilnadu, INDIA
}

\begin{abstract}
The endlessly increasing population in Asian nations such as India demands for the expeditious improvement in food production technology. Indian economy is predominantly based on agriculture. While farming, some important soil parameters such as ph, moisture, wetness and temperature square measure measured for obtaining high yield from soil. The method which is employed for measuring of those parameters is totally supported chemical action on soil sample. The process is usually distributed in close to Agriculture workplace. Soil Parameter Monitoring with Automatic Irrigation System include the measurement of these parameters on the field so that the farmer doesn't need to go somewhere else. This system also consists of a fully automated irrigation system which will turn on and off a pump as per the extent of wetness in soil. A traditional approach to measure these factors in AN agricultural atmosphere meant people manually taking measurements and checking them at various times. This report investigates a remote monitoring system using $\mathrm{HC}-05$ /HC05 Wifi Module for Arduino. These nodes collect and send data wirelessly to a centralized server, which collects the data, stores it and will allow it to be analyzed then displayed as needed and can also be sent to the client mobile. Also, a weather monitoring system can be integrated with this model which keep the farmer notified about the present climatic changes, so that the threshold of soil parameters for any particular action on the soil can be managed accordingly. We can also manage the irrigation based on the season i.e. winter, summer etc.
\end{abstract}

Keywords: IoT, Sensors, Microcontroller, Wi-Fi

\section{INTRODUCTION}

As the main aim of this project is to counter various serious issues through IoT. The primary focus of this project is to provide the real-time information of different soil parameters to the user along with the facility of irrigating the soil labor free by initiating the water pump through the user device. This product in all ways help to counter the following issues:

Deforestation: Due to improper maintenance of parks, trees on the road sides by the municipality due to less work force or scarcity of water. This product provides with the efficient watering system i.e. watering only if the soil moisture level drop down through certain range and not if not needed. This definitely helps in efficient use of resources as well as the work force
Preservation of resources: Improper irrigation by the farmers such as watering in abundance when water is provided by the municipality so that the soil moisture does not drop to a certain level. Every crop possess a certain minimum soil moisture threshold which must be checked but still no maximum limit is decided which leads in watering of soil in abundance by the farmers, which is not the efficient way to counter the problem. This project counters this problem by efficient irrigation of soil such there is no scarcity as well as soil does not possess high moisture content

Loss of rivers like Cauvery: There are basically two types of origination of rivers. First is the ice-fed rivers, which are fed

by the glaciers and are drastically affected by the improper usage and maintenance of resources and the by-product of it. Second are the forest-fed, which are basically fed by the ground water and in a way by the rainfall.

In case of maintaining both the types of rivers forestation plays a major role:

First to control carbon emission which plays a major role in depletion of ice-fed rivers. In today's world due to heavy electronic usage and maintaining huge datacenters lead to emission of carbon oxides which affects the environment in all possible negative ways.

Secondly helping the soil to hold rain-water is very important for the forest-fed rivers like Cauvery. Extreme and hot weather conditions lead to the evaporation of ground water which in turn depletes the rivers in a big way.

So eradicate these problems, proper plantation is very important that means the plant planted must be look after and nurtured fully so that it helps in the reduction of the same. This product provides the user with the overall parameters of the plantation soil and notifies the user before any soil content touches its threshold.

Apart from the use cases of the project, this prototype make sure to possess cost efficient system design, proper resource management architecture and smart use of technology for the Digital India.

\section{LITERATURE SURVEY}

Automatic Irrigation System For Agriculture Using WSN, Web based intelligent irrigation system is solution for this 


\section{International Journal of Engineering Applied Sciences and Technology, 2019 Vol. 4, Issue 6, ISSN No. 2455-2143, Pages 138-143 \\ Published Online October 2019 in IJEAST (http://www.ijeast.com)}

problem. It is automated and micro-controlled based can be control from remote location. It takes decision on sensor value of agriculture farm. Successful use of the wireless sensor technology what was able to automate the process, Microcontroller acts according to the control algorithm. Sensor out showing is analog in nature in the range of $0-5 \mathrm{v}$. Microcontroller converts analog data to digital data. After implementing this prototype, we can improve the traditional way of agriculture watering system in different region of India. Hence the project was successful in its objectives.

Automated Smart Irrigation System using Raspberry Pi, Smart water sprinkler irrigation system makes an efficient use of water for the growth of plants. Heart of the system is Raspberry Pi 3 mini computer, shown in figure 1. RaspberryPi has three devoted general purpose input outputs. In this work, webcam is interfaced with Raspberry Pi 3 via Wi-Fi Module. Raspberry $\mathrm{Pi}$ is the heart of the overall existing system. The Raspberry Pi Model 3 incorporates a number of enhancements and new features. Improved power expenditure, inflamed connectivity and greater IO are among the improvements to this powerful, small and lightweight. Soil moisture sensor gives a resistance variation at the output. That signal is applied to the comparator and signal conditioning circuit.

Localized Weather Monitoring System, Weather prediction is done by extensive analysis of data that is collected over a period of time. The climatic data of a meticulous location has a range of attributes like - temperature, pressure, wind, sunlight, rain. All these factors and their intermediaries contribute to the prediction of the climate under consideration. To reduce complexity we would build a mini weather station on top of every building there is in the city. Presume, if there are 1 million building, there will be 1 million small climate stations incorporated on the top just like a Tata Sky antenna is installed. This would help in collecting data from each area of the city, An algorithm will be used to learn each locations weather and its neighbouring locations dependencies which will help in currently identifying the climate. Thus people of that area can make plans accordingly depending on the weather predicted by the mini stations.

Automatic Irrigation System on Sensing Soil Moisture Content, Continuous increasing demand of food requires the control in highly specialized greenhouse vegetable rapid improvement in food production technology. Water is can be wholly rectified if we use automatic micro supplied frequently, often daily to maintain favorable soil controller based drip irrigation system. This automated irrigation brings into play an Arduino board ATmega328 micro-controller, is programmed to collect the input signal of changeable moisture circumstances of the earth via moisture detecting system.

Wireless Network Based Automatic Irrigation System, Using the automated irrigation system we can prove that the use of water can be reduced for different agricultural production. The irrigation system provide only requisite amount of water to crop. The automated irrigation system implemented is very feasible and cost effective. The system is very economical in terms of hardware component and power consumption. The system helps in saving of water and electricity. It can be implemented in large agricultural areas. With the help of GSM user can control the motor from anywhere.

Implementation of Weather Monitoring System, Meteorological parameters are measured by using an automated weather station using sensors without intervention of humans. The data is stored in a data logger. The recorded data can be physically downloaded to a computer at a later time for further processing. The communiqué system is a crucial element in an automated weather station. The Arduino Uno board is connected to the CPU by using data cable. DHT 11 temperature and humidity sensor is placed on the bread board. The sensor is again connected to the Arduino Uno board by using connecting wires which is a serial communication. Then upload the required program in Arduino Software (IDE) and the temperature and humidity readings are displayed on the serial monitor which are shown in the screen shots below. The environmental monitoring data sensors monitor the temperature and humidity.

Solar Driven Arduino Based Automatic Irrigation Using GSM, This problem can be perfectly rectified if we use automatic arduino based irrigation system in which the irrigation will take place only when there will be intense requirement of water. In this planned system, the wetness and temperature of plants are precisely controlled. Irrigation arrangement uses valves to revolve irrigation $\mathrm{ON}$ and $\mathrm{OFF}$. In this proposed hardware system, the driving element will be aurdino board. Soil moisture sensor and temperature sensor will be buried under the soil. This sensor senses and analyses the moisture content and temperature of the soil. The sensed data will the important play role in this proposed system and this will be sent to aurdino board. The sensed data about temperature and moisture content of soil will be displayed on the LCD screen. The information about supplying the water to the field will be sent to the farmer mobile phone via GSM concept.

GSM Based Automatic Irrigation Control System For Efficient Use Of Recourses And Crop Planning Using Mobile, The user communicate through SMS. The unit communicates with the system through GSM with the help of SIM card.GSM sends through continuously receives some node to another node. At introduced GSM SMS remote measurements and control green house data based system with base station. Base station is developed by microcontroller, GSM module, sensor and actuator. In this proposed hardware system, the driving element will be arduino board. Soil moisture sensor and temperature sensor will be buried under the soil. This sensor senses and analyses the moisture content and temperature of the soil. The sensed data will the important play role in this proposed system and this will be sent to arduino board. The sensed data about temperature and moisture content of soil will be displayed on the LCD screen. The information about supplying the water to the field will be sent to the farmer mobile. Weather Monitoring Station, Weather monitoring plays a very important role in human life hence study of weather system is necessary. At this time there are two types of the weather monitoring stations 


\section{International Journal of Engineering Applied Sciences and Technology, 2019 \\ Vol. 4, Issue 6, ISSN No. 2455-2143, Pages 138-143 \\ Published Online October 2019 in IJEAST (http://www.ijeast.com)}

available i.e. wired and wireless. Wireless arrangement has a little advantages more than the wired one hence popular now a days. Wireless knowledge is quickly increases and also used to watch weather parameters remotely. Instead of analog instruments now day we can use with internally calibrated. In adding together with above all sort sense time is very less for this reason digital method is advantageous. The coordination design consist of mast as well as receiver. Transmitter section consist of different types of sensing units such as temperature measurement, Humidity measurement, Atmospheric pressure measurement, Rainfall measurement, Wind speed and wind direction measurement.

\section{PROPOSED WORK}

The system that we present here is the amalgamation of IOT based software and hardware apparatus. The software part consist of Arduino IDE(1.8.10) along with the Losant cloud services. Hardware components where the various sensors that were used along with the microcontroller and a DC motor to pump the water. Losant provides us with an online platform to display the results and we can also get the Alerts in our smart phones.

Figure shows the design of the project. The Losant platform is connected to microcontroller through Http protocol and the later is connected to the smart phone via MQTT protocol. The motor is controlled by the threshold set in the code and at the same time an alert message is sent to the mobile phone.
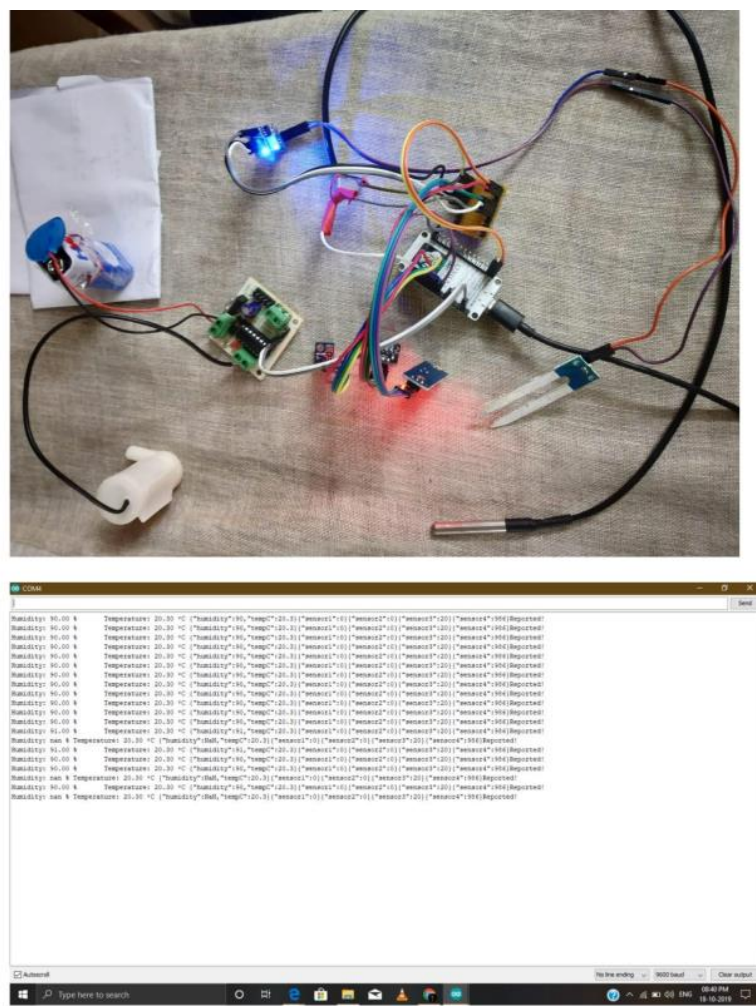

\section{METHODOLOGY}

This proposed method to implement the system has various sections:

In the outdoor section, various sensors are deployed in the field like temperature sensor, soil moisture sensor, humidity sensor, pressure sensor and light sensor. The data collected from these sensors are transmitted to the microcontroller (NODE MCU) through RS232.

In control section, the received data is verified with the threshold values, we have set up some certain threshold for each of the sensors. If the data exceeds the threshold value it shows the graphical representation of the same in the dashboard that we have created in the Losant platform further an Alert is also sent to the smart phone in real time. This alarm is transmitted as a memorandum to the user(farmer) and then without human intervention the power is switched OFF after performing the task. The values are generated in the dashboard and the farmer gets the detailed description of the values. Our system works in complete automatic mode, the microcontroller controlled motor gets switched ON and OFF automatically if the value exceeds the threshold point. As soon as the microcontroller (NODE MUC) is started, the Losant platform becomes active and starts representing the values in a refresh interval of 5 seconds. This is achieved by sending data to Losant cloud through the WIFI module.

The Losant platform is connected to microcontroller through Http protocol and the later is connected to the smart phone via MQTT protocol. Erstwhile factors like the temperature, environmental humidity, soil moisture and the pressure sensors shows the threshold value and the water level sensor is used just to indicate the level of water inside field or the water resource.

In addition to this we have deployed the pressure sensor and the atmospheric temperature and moisture sensor to calculate the most appropriate weather condition the vicinity of the setup. We have set the pre existing set pair of values that predict the weather pattern that will in hand help the farmers in their daily day to day activities and it will improve the overall efficiency of the system.

\section{HARDWARE USED}

\section{Soil Moisture Sensor:}

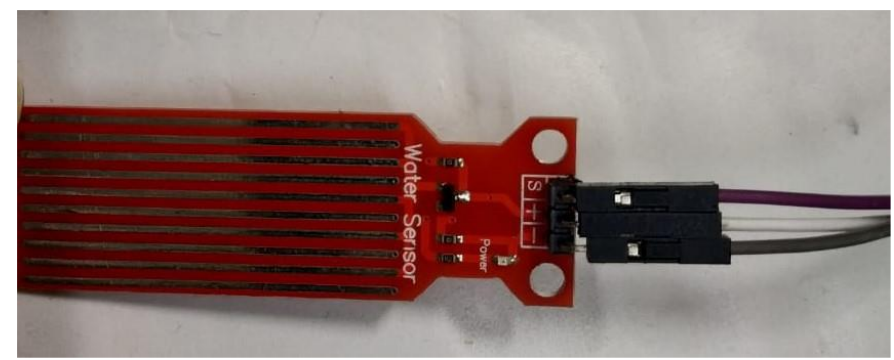

This Capacitive Soil Moisture Sensor V1.2 measures soil moisture levels by capacitive sensing rather than resistive sensing like other sensors on the market. It is made of corrosion resistant material which gives it an excellent service life. 


\section{International Journal of Engineering Applied Sciences and Technology, 2019 \\ Vol. 4, Issue 6, ISSN No. 2455-2143, Pages 138-143 \\ Published Online October 2019 in IJEAST (http://www.ijeast.com)}

This module includes an onboard voltage regulator which gives it an operating voltage range of $3.3 \sim 5.5 \mathrm{~V}$. It is perfect for low-voltage MCUs, both $3.3 \mathrm{~V}$, and $5 \mathrm{~V}$. For compatibility with a Raspberry Pi, it will need an ADC converter. This soil moisture sensor is compatible with our 3-pin "Gravity" interface, which can be directly connected to the Gravity I/O expansion shield.

\section{Soil Temperature Sensor:}

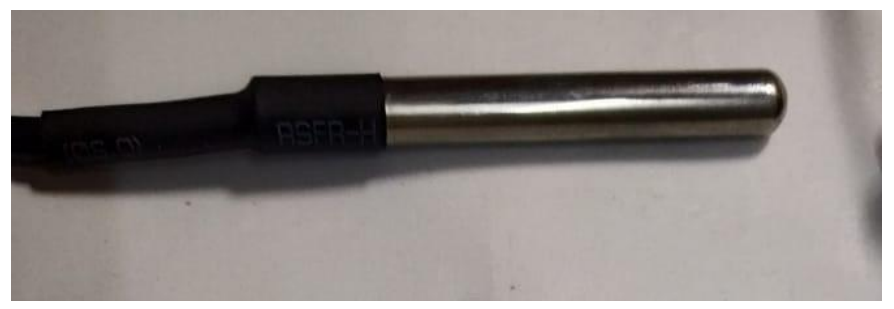

Platinum resistance thermometers (PRTs) offer excellent accuracy over a wide temperature range (from -200 to +850 $\left.{ }^{\circ} \mathrm{C}\right)$. Standard sensors are are available from many manufacturers with various accuracy specifications and numerous packaging options to suit most applications. Unlike thermocouples, it is not necessary to use special cables to connect to the sensor.

\section{Air Humidity and Temperature Sensor:}

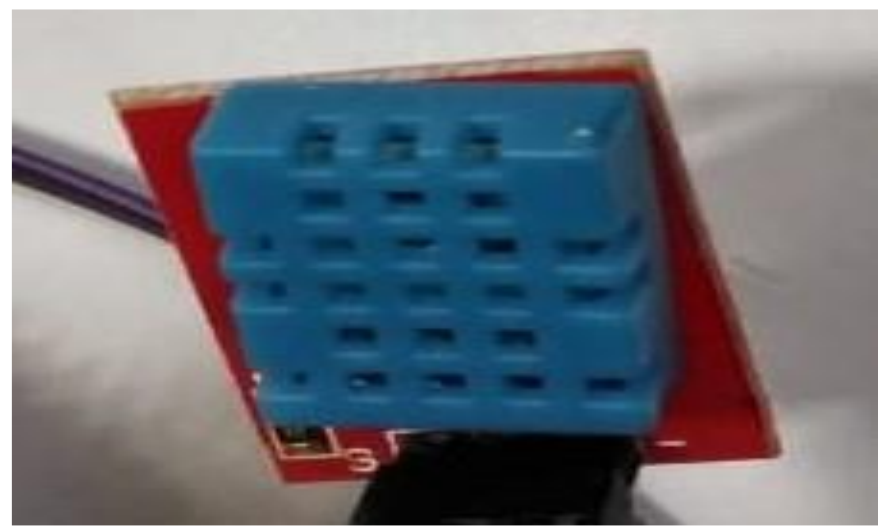

DHT temperature \& humidity sensors: The low cost DHT temperature \& humidity sensors. These sensors are very basic and slow, but are great for hobbyists who want to do some basic data logging. The DHT sensors are made of two parts, a capacitive humidity sensor and a thermistor. There is also a very basic chip inside that does some analog to digital conversion and spits out a digital signal with the temperature

and humidity. The digital signal is fairly easy to read using any microcontroller.

Node mcu:

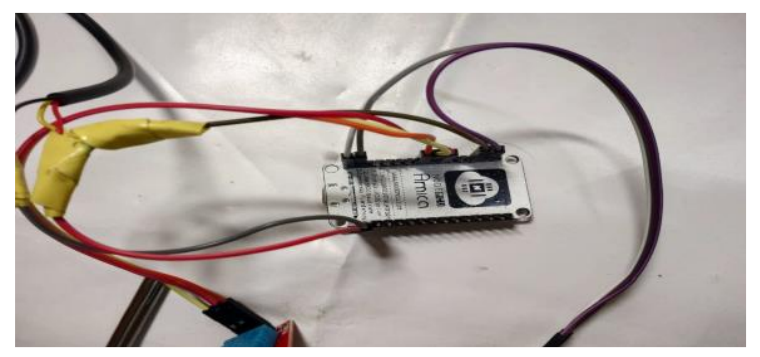

Node mcu is an open-source firmware and development kit that helps you to prototype or build IoT product. Node mcu too like Arduino Uno can be programmed directly using Arduino IDE. Node mcu has 10 digital pins and only one analog pin. Communication Interface: The user must connect to the system using Node mcu that is to gain control and a dashboard to display the results will be made currently we will be using through wifi module of the Node mcu.

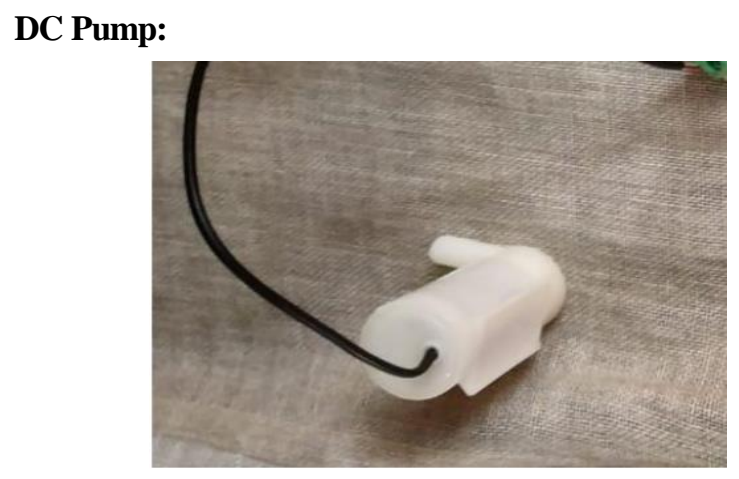

DC powered pumps use direct current from motor, battery, or solar power to move fluid in a variety of ways. Motorized pumps typically operate on $6,12,24$, or 32 volts of DC power.

\section{SOFTWARE USED}

\section{Arduino IDE (1.8.10):}

The Arduino Integrated Development Environment (IDE) is a cross-platform application (for Windows, macOS, Linux) that is written in the programming language Java. It is used to write and upload programs to Arduino compatible boards, but also, with the help of 3rd party cores, other vendor development boards.

\section{Losant cloud (Platform provider for IoT systems):}

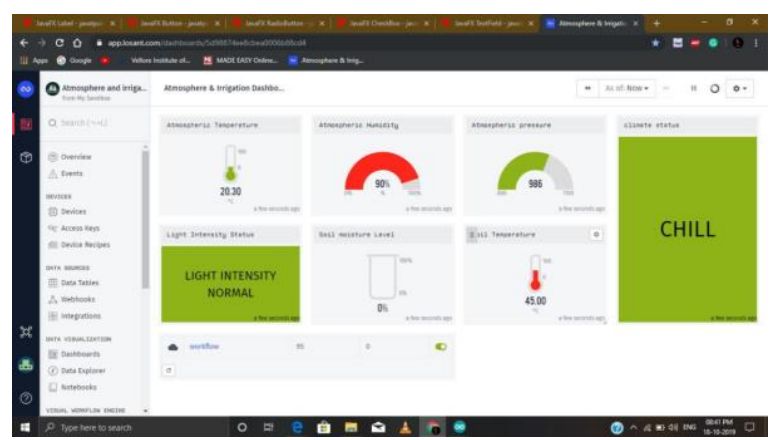




\section{International Journal of Engineering Applied Sciences and Technology, 2019 \\ Vol. 4, Issue 6, ISSN No. 2455-2143, Pages 138-143 \\ Published Online October 2019 in IJEAST (http://www.ijeast.com)}

The Losant IoT Platform is an application enablement platform which allows enterprises to effectively build applications that securely scale to millions of devices. With real-time stream processing and batch processing capabilities, users can create dynamic experiences and perform complex analytics.

\section{EXPERIMENTATION AND RESULTS.}

The output shown below denotes the temperature, soil moisture condition and the intruder detection. The second result is the output from the Android Application that is developed in the mobile phone. It determines the temperature, humidity, moisture and air parameter detection
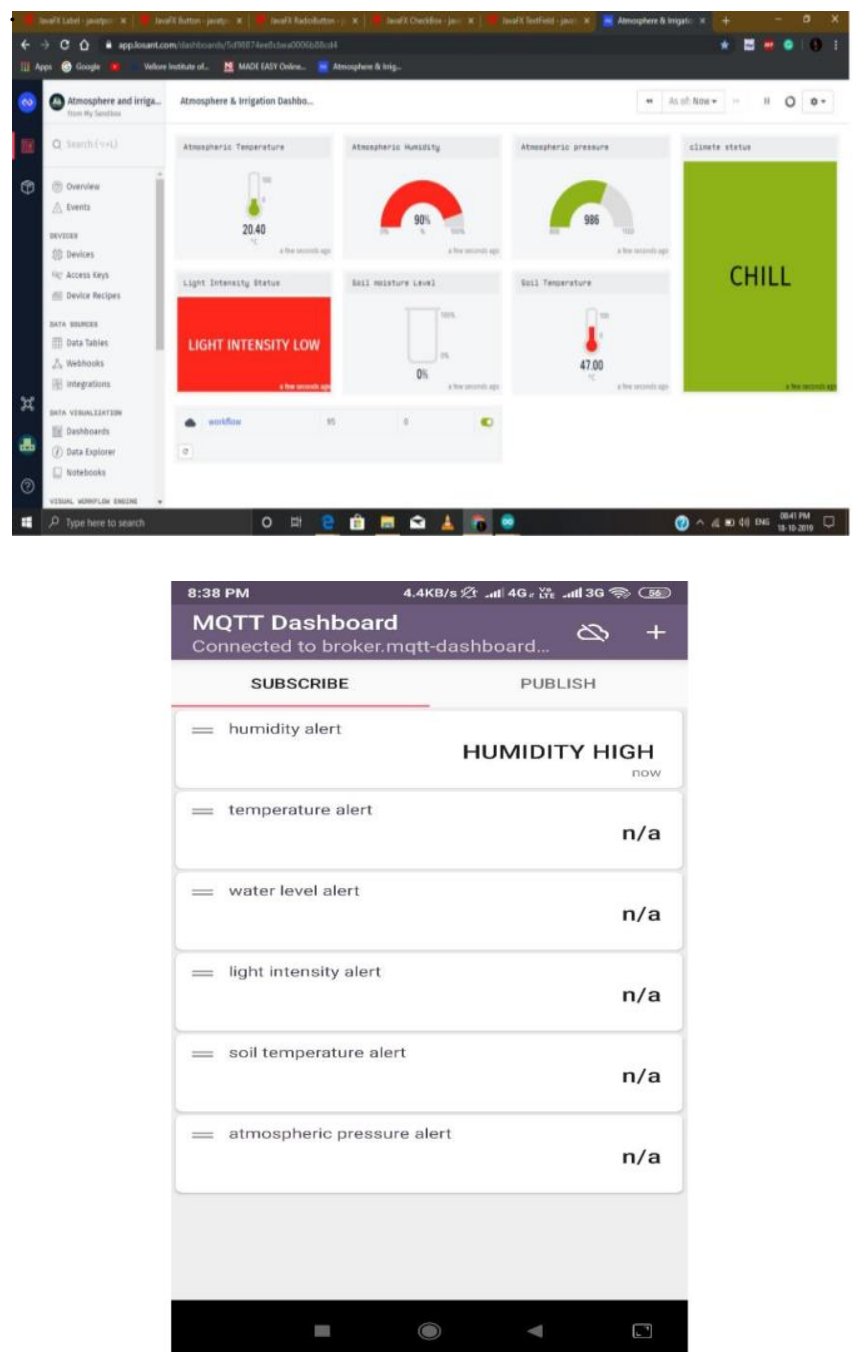

VIII. CONCLUSION

We have successfully built a proper fully functioning working model of the automatic soil irrigation System. Our model takes soil parameters like moisture, wetness, temperature and weather parameters like humidity, temperature into consideration to make output more accurate. Our model has a user friendly interface which allows farmer to change threshold values of different factors. This enables farmer to customize model to the type of crop, weather and landscape. We are utilizing features of cloud computing to store, process and monitor data. This is a very helpful substitute for an actual visit to irrigation department office. This model also has an actuator, water pump making it an automated irrigation system. And as for the future developments this system can be improved by developing this system for large acres of terrain.

\section{REFERENCES}

[1] K.Lakshmisudha, Swathi Hegde, Neha Kale, Shruti Iyer, (July 2011) “ Smart Precision Based Agriculture Using Sensors", International Journal of Computer Applications (0975- 8887), (Volume 146-No.11)

[2] Nikesh Gondchawar, Dr. R.S.Kawitkar(June 2016). "IoT Based Smart Agriculture", International Journal of Advanced Research in Computer and Communication Engineering (IJARCCE), (Vol.5, Issue 6)

[3] M.K.Gayatri, J.Jayasakthi, Dr.G.S.Anandhamala,(2015). "Providing Smart Agriculture Solutions to Farmers for Better Yielding Using IoT", IEEE International Conference on Technological Innovations in ICT for Agriculture and Rural Development.

[4] Chetan Dwarkani M, Ganesh Ram R, Jagannathan S, R. Priyatharshini, (TIAR 2015). "Smart Farming System Using Sensors for Agricultural Task Automation", IEEE International Conference on Technological Innovations in ICT for Agriculture and Rural Development.

[5] S. R. Nandurkar, V. R. Thool, R. C. Thool, (2014) "Design and Development of Precision Agriculture System Using Wireless Sensor Network", IEEE International Conference on Automation, Control, Energy and Systems (ACES),

[6] Joaquín Gutiérrez, Juan Francisco Villa-Medina, Alejandra Nieto-Garibay, and Miguel Ángel PortaGándara,(2013) "Automated Irrigation System Using a Wireless Sensor Network and GPRS Module", IEEE Transactions on Instrumentation and Measurements, (0018-9456)

[7] Dr. V .Vidya Devi,G. Meena Kumari, (2013) "RealTime Automation and Monitoring System for Modernized Agriculture" ,International Journal of Review and Research in Applied Sciences and Engineering (IJRRASE) Vol3 No.1. (PP 7-12).

[8] Meonghun Lee, Jeonghwan Hwang, Hyun Yoe, (2013) "Agricultural Protection System Based on IoT", IEEE $16^{\text {th }}$ International Conference on Computational Science and Engineering.

[9] Monika Jhuria, Ashwani Kumar, Rushikesh Borse, ,(2013) "Image Processing for Smart Farming: Detection of Disease and Fruit Grading", IEEE Second International Conference on Image Information Processing (ICIIP). 


\section{International Journal of Engineering Applied Sciences and Technology, 2019 \\ Vol. 4, Issue 6, ISSN No. 2455-2143, Pages 138-143 \\ Published Online October 2019 in IJEAST (http://www.ijeast.com)}

[10] Orazio Mirabella and Michele Brischetto, (2011) "A Hybrid Wired/Wireless Networking Infrastructure for Greenhouse Management", IEEE Transactions on Instrumentation and Measurement, vol. 60, no. 2, (pp 398- 407).

[11] C. Liu, W. Ren, B. Zhang, and C. Lv,(2011)“The application of soil temperature measurement by $\operatorname{lm} 35$ temperature sensors," International Conference on Electronic and Mechanical Engineering and Information Technology, vol. 88, no. 1,(pp. 1825-1828) .

[12] D.D.Chaudhary1, S.P.Nayse2, L.M.Waghmare, (February 2011) "Application of wireless sensor networks for greenhouse parameter control in precision agriculture", International Journal of Wireless \& Mobile Networks (IJWMN) Vol. 3, No. 1.

[13] Q. Wang, A. Terzis and A. Szalay, (2010) "A Novel Soil Measuring Wireless Sensor Network", IEEE Transactions on Instrumentation and Measurement, (pp. 412-415).

[14] Ji-woong Lee, Changsun Shin, Hyun Yoe, (July, 2010) "An Implementation of Paprika Green house System Using Wireless Sensor Networks", International Journal of Smart Home Vol.4, No.3.

[15] Mahesh M. Galgalikar, (2010)"Real-Time Automization Of Agricultural Environment for Social Modernization of Indian Agricultural System", IEEE.

[16] Y. Song, J. Wang, X. Qiao, W. Zheng, and X. Zhang, (2010) "Development of multi-functional soil temperature measuring instrument," Journal of Agricul- tural Mechanization Research, vol. 9, no. 1,( pp. 80-84). 